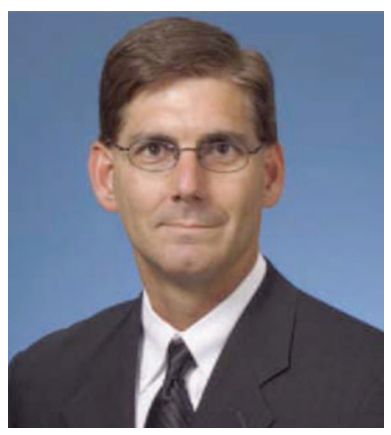

\title{
Working in the Conference Halls
}

I always enjoy going to the SPIE Defense, Security, and Sensing Conference and the SPIE Security and Defence Conference. The latter was held in Prague this past September and I spent as much time in the hallway as I did listening to technical papers. While the papers this year were very good, I felt like the time I spent with colleagues was just as valuable, if not more important.

Just to give you some examples on the value of spending time in the conference hallway, consider the following. I caught up with Eddie Jacobs, the Acting Chairman of the Electrical Engineering Department at the University of Memphis. He used to work for me as a Branch Head when I was a Division Head at Army Night Vision Laboratory. We discussed imaging with sparse detector arrays and the performance of imaging in underwater systems. Norm Kopeika from Ben Gurion University in Israel and I worked on imaging through turbulence many years ago and he described some new detectors for imaging in the millimeter-wave region. During our discussion, he invited me to Ben Gurion to give a talk on infrared system performance research. I caught up with Arie DeJong from the Netherlands Physics and Electronics Laboratory. We used to belong to the same NATO technical group and he is an expert in the area of infrared search and track systems. He had taken some maritime imaging data in Africa and was excited about the results. Eustace Dereniak and I discussed how small infrared detectors can be produced and still provide good performance. Stace will be the President of SPIE next year. He talked about how he is excited about it and how it is going well so far.
Just to show how small the electro-optics community is, I sat in the same laboratory next to a post-doc from Spain, Javier Alda, when I was working on my PhD at the University of Central Florida. We became close friends, and he and his wife would come over for dinner. Ten years later, I was assigned to a NATO technical committee and his doctoral student Jose Lopez was also assigned to the same committee. Some eight or nine years later, his graduate student Rodrigo Linares was assigned to a different committee where I was already member. Anyway, I missed them this year in the conference hallway as I always enjoy talking to at least one of them to see how the others are doing. This small group of researchers has convinced me if I were 20 years old again, I would study electro-optics in Spain by day and learn how to flamenco dance at night.

So, the message this month is to spend time in the conference hallway. The information you gain from old friends and colleagues could be the most important content of the conference. The discussions with colleagues can provide validation of your research approach, provide new research ideas, result in new applications, and be a very enjoyable experience. The message for bench scientists and engineers is not to hibernate in your laboratory and to get out and talk to colleagues and make new connections. These interactions lead to more opportunities, better research, new ideas, and a more enjoyable career when you have others to share it with.

Ronald G. Driggers Editor 